\title{
Screening for atrial fibrillation
}

\section{Zhenisgul Tlegenova $^{1}$, Gulmira Kudaiberdieva ${ }^{2}$, Bekbolat Zholdin' ${ }^{1}$, Ayan Abdrakhmanov ${ }^{3}$}

${ }^{I}$ No 2 Department of Internal Medicine, Marat Ospanov West Kazakhstan State Medical University, Aktobe, Republic of Kazakhstan

${ }^{2}$ Center of Postgraduate Education and Research, Bishkek, Kyrgyzstan;

${ }^{3}$ Interventional Arrhythmology Department, National Research Cardiac Surgery Center, Astana, Republic of Kazakhstan

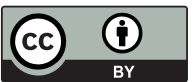

This work is licensed under a Creative Commons Attribution 4.0 International License

\section{Received: 29-11-2017}

Accepted: 22-12-2017

UDC: 616.6

\section{J Clin Med Kaz 2018;1(47):18-24}

Corresponding Author: Zhenisgul Tlegenova, Marat Ospanov West Kazakhstan State Medical University. Adress: 68, Maressyev Sstreet, 030019, Aktobe, Republic of Kazakhstan.

Tel.:+77074998565 E-mail: Tlegenova_G@mail.ru

\section{Abstract}

Atrial fibrillation is the most common arrhythmia that physicians face in their clinical practice. Atrial fibrillation is associated with a higher risk of thromboembolic events, all-cause mortality, frequent hospitalizations, decreased quality of life. Early detection of atrial fibrillation is important for optimal management and improvement the prognosis of atrial fibrillation patients. Evidence on new methods of atrial fibrillation screening discussed in this review, allows considering them more accurate than simple pulse palpation.

Key words: atrial fibrillation, stroke, screening, electrocardiogram

\section{ЖҮРЕКШЕЛЕР ЖЫБЫРЫНА АРНАЛҒАН СКРИНИНГ}

Тілегенова Ж.Ш. ${ }^{1}$, Құдайбердиева Г.3. ${ }^{2}$, Жолдин Б.К. ${ }^{1}$, Абдрахманов А.С. ${ }^{3}$

ํ№2 Ішкі аурулар кафедрасы, Марат Оспанов атындағы Батыс Қазақстан Мемлекеттік медицина университеті, Ақтөбе, Қазақстан Республикасы

²Жоғары оқу орнынан кейінгі білім беру және ғылым орталығы, Бішкек Қырғызстан;

${ }^{3}$ Интервенциялық аритмология бөлімшесі, Ұлттық ғылыми кардиохирургия орталығы, Астана, Қазақстан Республикасы

\section{ТҰЖЫРЫМДАМА}

Жүрекшелер жыбыры - дәрігер тәжірибесінде ең жиі кездесетің ырғақ бұзылысы. Жүрекшелер жыбыры клиникалық салдары тромбоэмболиялық асқыну, жүрек жетіспеушілігі, кенеттен болатын өлім, жиі госпитализация, өмір сапасының төмендеуі болып табылады. Жүрекшелер жыбыры неғұрлым ерте анықтау тиімді ем тағайындауға және қолайсыз асқынулар жиілігін азайтуға мүмкіндік береді. Бұл шолуда пульс пальпациясына қарағанда нақты болып табылатын жүрекшелер жыбыры скринингінің жаңа әдістері туралы дәлелді медицинаның мәліметтері берілген.

Негізгі сөздер: жүрекшелер жыбыры, скрининг, ишемиялық инсульт,электрокардиограмма

\section{СКРИНИНГ НА ФИБРИЛЛЯЦИЮ ПРЕДСЕРДИЙ}

Тлегенова Ж.Ш.. ${ }^{1}$, Кудайбердиева Г.3. ${ }^{2}$, Жолдин Б.К. ${ }^{1}$, Абдрахманов А.С. ${ }^{3}$

${ }^{1}$ Кафедра внутренних болезней № 2, Западно-Казахстанский Государственный медицинский университет имени Марата Оспанова, Актобе, Республика Казахстан

${ }^{2}$ Центр последипломного образования и науки, Бишкек, Кыргызстан;

${ }^{3}$ Отделение интервенционной аритмологии, Национальный научный кардиохирургический центр, Астана, Республика Казахстан

\section{PEЗЮME}

Фибрилляция предсердий - самая распространенная аритмия в практике врача. Клинические последствия фибрилляции предсердий включают тромбоэмболические осложнения, сердечную недостаточность, преждевременную смертность, частые госпитализации, низкое качество жизни. Раннее обнаружение фибрилляции предсердий позволит назначить эффективную терапию и уменьшить частоту неблагоприятных исходов. В данном обзоре представлены данные доказательной медицины о новых методах скрининга фрибрилляция предсердий, которые являются более точными, чем пальпация пульса.

Ключевые слова: фибрилляция предсердий, ишемический инсульт, скрининг, электрокардиограмма 


\section{Введение}

Фибрилляция предсердий (ФП) является самой распространенной аритмией в практике врача, ею страдает от 30 до 100 миллионов человек в мире. В ближайшее время количество больных увеличится из-за широкой распространенности факторов риска развития ФП, к которым относятся артериальная гипертензия (АГ), пожилой возраст, сахарный диабет (СД), ожирение [1,2].

Клинические последствия ФП включают тромбоэмболические осложнения (ТЭО), сердечную недостаточность, преждевременную смертность, частые госпитализации, низкое качество жизни [3].

В соответствии с существующими рекомендациями, раннее обнаружение ФП и назначение эффективной терапии уменьшает частоту неблагоприятных исходов [4]. В ряде исследований продемонстрировано, что больные с впервые выявленной ФП имеют высокий риск ишемического инсульта (ИИ) в 67-90\% случаев [5,6]. Своевременно начатое лечение антикоагулянтами снижает риск ИИ на $67 \%$, а общую смертность на 26\% [7].

Трудно зарегистрировать бессимптомную ФП, так как больные не чувствуют симптомов аритмии и не обращаются к врачу и пароксизмальную ФП в связи с её кратковременным течением. По данным регистра EORP-AF до 40\% пациентов с ФП имеют бессимптомное течение аритмии [8].

Бессимптомное и пароксизмальное течение не делает ФП менее опасной [9]. Без скрининга выявить такое течение аритмии до развития грозных осложнений не представляется возможным. В недавнем обзоре Lis Neubeck и соавторов приводятся аргументы, что скрининг на ФП соответствует критериям ВОЗ Уильсон и Юнгер для программ скрининга [10]. Эффективность скрининга на ФП зависит от целевой популяции, точности диагностического теста и длительности регистрации ЭКГ [11].

Важными факторами риска развития ФП по данным Framingham Heart Study являются: возраст, сердечная недостаточность, мужской пол, наличие сердечного шума, ожирение, АГ, удлинение интервала PR [12]. B ARIC Study к развитию ФП предрасполагали курение, высокий рост, наличие СД, ишемической болезни сердца и гипертрофии левого желудочка [13].

В литературе есть указания, что с помощью шкалы CHA2DS2VASc можно прогнозировать пациентов с высоким риском развития ФП[14].

Высокая распространенность ФП и её осложнений, приводящих к потере трудоспособности, обосновывает поиск и разработку методов раннего выявления этой аритмии, которая, как указано выше, часто носит бессимптомный и пароксизмальный характер.

Цель настоящего обзора - представить современные методы и стратегии скрининга на ФП.

\section{Устройства для скрининга на фибрилляцию предсердий}

B систематическом обзоре Taggar JS и соавторы сравнили устройства для скрининга на ФП [15]. Все тесты были разделены на 4 категории: пальпация пульса, прибор для измерения артериального давления(АД) с алгоритмом обнаружения ФП, портативные одноканальные ЭКГ регистраторы, смартфоны с возможностью записи одного канала ЭКГ. на ФП.

На Рисунке 1 представлены устройства для скрининга

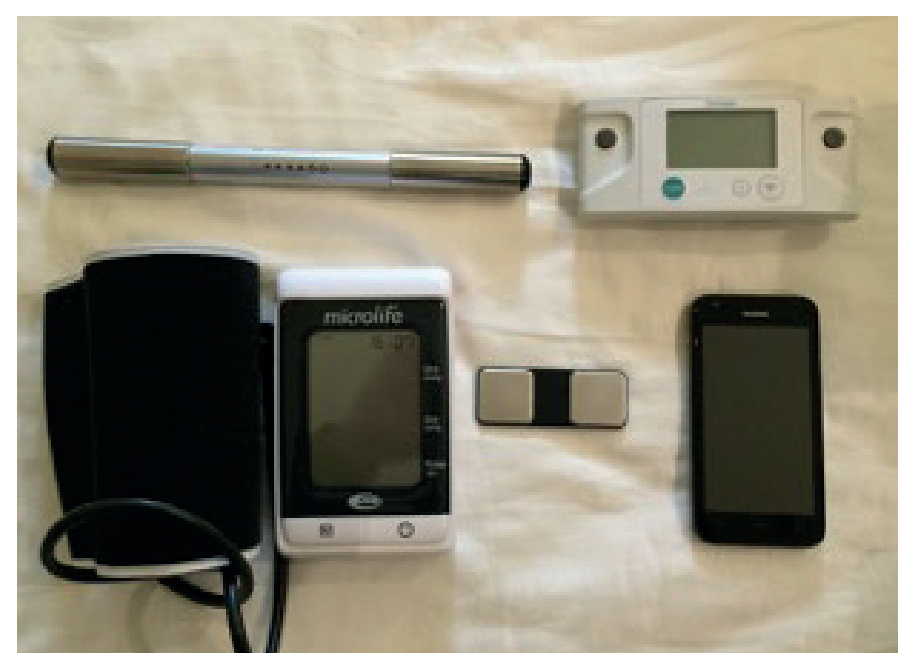

Рисунок 1 - Портативные устройства MyDiagnostick, Aliver Cor, Zenicor ECG для записи одного канала ЭКГ, тонометр ВР Ноmе-А

Простейшим методом обнаружения ФП является пальпация пульса. Скрининг при этом проходит в 2 этапа. Вначале обнаруживают пациентов с нерегулярным пульсом, на следующем этапе записывают ЭКГ для подтверждения ФП. ФП подтверждается регистрацией участка ЭКГ продолжительностью $\geq 30$ сек с нерегулярностью интервалов RR и отсутствием зубцов P [16]. Авторы вышеприведенного систематического обзора пришли к заключению, что пальпация пульса обладает сопоставимой чувствительностью с другими тестами от $87 \%$ до 97\%, но низкой специфичностью от $70 \%$ до $81 \%$. Это приводит к большому количеству ложноположительных результатов.

В 2013 году Британский национальный институт здоровья NICE установил, что скрининг на ФП с использованием устройства для осциллометрического измерения АД (Watch ВР Ноme-A) с алгоритмом обнаружения ФП является экономическим выгодным [17]. Скрининг на ФП стали проводить в учреждениях первичной медико-санитарной помощи во время офисного измерения АД у пациентов $\geq 65$ лет. ФП, которую обнаружил данный прибор, необходимо подтвердить записью ЭКГ.

В настоящее время доступны недорогие портативные приборы для регистрации одного канала ЭКГ. В одних моделях электроды, прикладываются к груди, в других моделях пальцы рук прикладываются к сенсорным кнопкам устройства. Непрерывная запись осуществляется за 30-60 секунд и передается через мобильный телефон или Wi-Fi в аналитический центр. Простота использования устройств, отсутствие электродов на теле повышает приверженность пациентов к методике и позволяет проводить длительное мониторирование.

В исследованиях проводилась, как однократная запись 30 секунд, так и интермиттирующая запись по 30 сек дважды в день в течение 14-30 дней. Интермиттирующая запись была в 2-3 раза эффективнее [18]. В исследовании STROKESTOP было показано, что 50\% опрошенных участников в возрасте 75-76 лет могут пользоваться портативными устройствами для скрининга ФП.

По мнению исследователей необходимо отдавать предпочтение приборам с автоматическим алгоритмом обнаружения ФП, который сможет отличить синусовый ритм от суправентрикулярных аритмий, включая ФП [19].

На Рисунке 2 представлена запись ЭКГ с синусовым ритмом и с ФП с помощью устройства MyDiagnostick. 

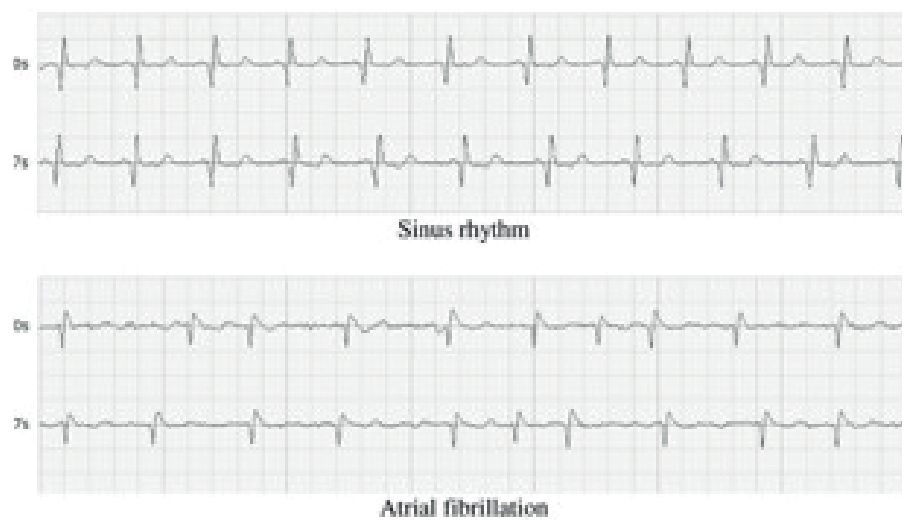

Рисунок 2 - Запись ЭКГ с синусовым ритмом и фибрилляцией предсердий с помощью устройства MyDiagnostick

Одноразовые патчи записывают ЭКГ до 14 дней. Установлено, что они более чувствительны, чем 24- часовое мониторирование по Холтеру в обнаружении ФП[20].

Повсеместное использование смартфонов и существование специальных приложений к ним позволило использовать эти гаджеты для записи ЭКГ. В данной технологии анализируется ритм, записанный с помощью приложения пальца на камеру смартфона для плетизмографии. Метод является привлекательным, так как нет необходимости в дополнительном оборудовании, нет ограничений в длительности записи, есть возможность передачи данных по сотовой сети. Однако, существуют трудности, связанные с артефактами записи, ограниченными диагностическими возможностями одноканальной ЭКГ, необходимостью передачи, хранения и интерпретации больших объемов информации, отсутствием данных о длительности ФП, отсутствием правил проверки смартфонов и приложений к ним [21].

Как видно из Таблицы 1, альтернативные методы обнаружения ФП: устройство для измерения АД, портативные регистраторы ЭКГ, смартфоны с возможностью регистрации ЭКГ являются более точными, чем пальпация пульса.

Таблица 1 Чувствительность и специфичность альтернативных методов обнаружения ФП и пальпации пульса (адаптировано из Mairesse $\mathrm{GH}$ et all, 2017г.)

\begin{tabular}{|l|l|l|}
\hline & Чувствительность & Специфичность \\
\hline Пальпация пульса & $87-97 \%$ & $70-81 \%$ \\
\hline $\begin{array}{l}\text { Прибор для } \\
\text { автоматического } \\
\text { измерения АД }\end{array}$ & $93-100 \%$ & $86-92 \%$ \\
\hline $\begin{array}{l}\text { Портативное устройство } \\
\text { для записи одного канала } \\
\text { ЭКГ }\end{array}$ & $94-98 \%$ & $76-95 \%$ \\
\hline Смартфон & $98,5 \%$ & $91,4 \%$ \\
\hline
\end{tabular}

\section{Стратегии скрининга на фибрилляцию предсердий}

Различают систематический и оппортунистический скрининг на ФП. При оппортунистическом скрининге ФП устанавливают, когда пациент по разным причинам приходит за медицинской помощью, а врачи, используя случай, оценивают регулярность ритма методом пальпации пульса или устройством. При нерегулярности пульса пациента направляют на ЭКГ.
Систематический скрининг проводится в целевой группе, которая имеет высокий риск ФП. Это, прежде всего, возраст 75 лет и старше. Все пациенты из этой группы приглашаются на скрининг.

Эффективность оппортунистического скрининга на ФП изучалась в ряде исследований. В исследовании SEARCH- AF в период с 2012 по 2013 г скрининг ФП проводился с помощью устройств Aliver Cor (Aliver CorInc., SanFrancisco, USA) у 1000 посетителей аптек старше 65 лет. ФП была зарегистрирована у 1,5\% испытуемых, из них впервые выявленная ФП обнаружена в 1,0\% (10/1000) [22].

Висследовании КaasenbroodF и соавторовпользовались устройством MyDiagnostic (Applied Biomedical SystemBV, Maastricht, Netherlands) для скрининга у пациентов $\geq 65$ лет, обратившихся за сезонной вакцинацией. ФП была обнаружена у 1,1\% пациентов (37/3269) [23].

B исследовании Smyth и соавторов врачи общей практики пальпировали пульс у всех пациентов $\geq 65$ лет (медиана возраста 74 года) во время обычной консультации. Больного с нерегулярным пульсом направляли на ЭКГ. За 6 месяцев исследования ФП выявили у $0,8 \%$ обследованных [24].

Программа систематического скрининга использовалась в исследование STROKESTOP. У пациентов 75-76 лет с помощью ЭКГ- регистратора Zenicor - ECG (Zenicor Medical SystemAB, Стокгольм, Швеция) проводили запись ЭКГ по 30 сек дважды в день на протяжении 14 дней. Исследователи выявили новые случаи ФП у 218 (3\%) участников. С первой регистрации было обнаружено $16.6 \%$ эпизодов ФП. Большинство эпизодов ФП (83.4\%) были выявлены при повторных регистрациях ЭКГ.

В аналогичном исследовании 848 пациентам 75-76 лет делали стандартную ЭКГ. Если ФП не обнаруживали, но участник имел риск ИИ $\geq 2$ баллов по шкале CHA2DS2$\mathrm{VASc}$, то 14 дней проводили интермиттирующую запись ЭКГ с использованием портативного устройства. Новые случаи ФП выявили у 5,2\% участников [25].

В Бельгии с 2010г по 2014г. во время добровольных Национальных недель сердечного ритма, о которых объявлялось в средствах массовой информации, взрослому населению $\geq 18$ лет выполнялась одна 30 секундная запись ЭКГ с использованием портативного устройства MyDiagnostic. Новые случаи ФП выявлены у 1,1\% участников [26].

В рандомизированном контролируемом исследовании SAFE сравнили оппортунистический и систематический скрининг на ФП с обычной клинической практикой. Исследование было проведено в 50 центрах первичной медико-санитарной помощи в Англии и включало 14802 пациентов в возрасте $\geq 65$ лет (75,3 \pm 7,2 года). Пациенты были рандомизированы в 3 группы. В 1 группе проводили пальпацию пульса и при его нерегулярности записывали ЭКГ, во 2 группе всем больным проводилась регистрация одной ЭКГ, в 3 группе (контрольной) активный скрининг не проводился, а случаи ФП выявляли на основе клинических проявлений. Через 12 месяцев наблюдения новые случаи ФП были обнаружены чаще в группах скрининга у 1,63\%, чем в контрольной группе у $1,04 \%(p<0,05)$. Оба вида скрининга выявили аналогичное количество случаев ФП $(1,64 \%$ и $1,62 \%)$ [27].

Moran и соавторы провели мета-анализ с общим числом участников $(\mathrm{n}=122000)$. Ученые показали, что при однократном скрининге с использованием ЭКГ или пальпации пульса распространенность ФП у пациентов $\geq$ 
65 лет увеличивается с 2,3\% до 4,4\%, а выявление новых случаев ФП с 1,0\% увеличивается до 1,4\% [28].

Несмотря на одинаковую эффективность систематического и оппортунистического скрининга, следует отметить, что при систематическом скрининге отклик пациентов невысокий, так в исследовании STROKESTOP участвовало 7173 человек из 14387 (54\%) приглашенных; в исследовании Engdahl и соавторов ученые отправили приглашение 1330 пациентам, откликнулось только 848 (64\%) лиц.

\section{Скрининг на фрибрилляцию предсердий после перенесенного ишемического инсульта}

Важное клиническое значение имеет выявление ФП у пациентов с эмболическим инсультом неопределенного генеза (ESUS), поскольку у них при обнаружении ФП имеется, как минимум 2 балла по шкале CHA2DS2VASc. Tермин ESUS был предложен в 2014г. Hart RG и соавторами, чтобы выделить пациентов с криптогенным инсультом, у которых эмболия является наиболее вероятной причиной ИИ [29]. Причиной ESUS часто является пароксизмальная ФП. Источником эмболии может быть левое предсердие, левый желудочек, восходящая аорта, каротидные артерии.

На стандартной ЭКГ при поступлении в стационар ФП имеют 18,8\% пациентов с инсультом. Дополнительные ЭКГ в период госпитализации и Холтеровское мониторирование выявляют дополнительно 2,26\% пациентов с ФП [30]. Семисуточное мониторирование повышает обнаружение ФП до 12,5\% [31]. Информативными являются первые трое суток, а у трети пациентов исследование не завершается по причине отсоединения электродов.

«Регистраторы событий» активируются самим пациентом по заранее согласованному с лечащим врачом алгоритму (например, 30 сек дважды за день утром и вечером).

В исследовании EMBRACE сравнивали частоту обнаружение ФП при 30 дневном мониторирование с помощью регистратора событий (группа вмешательства) и при традиционном 24 часовом амбулаторном мониторирование ЭКГ (контрольная группа) [32]. В группе длительного наблюдения ФП была обнаружена у 16,1\%, в контрольной группе только у $3,2 \%, \mathrm{p}<0,001$.

В рандомизированном проспективном исследовании «CRYSTAL AF» подкожный регистратор сердечного ритма был имплантирован 221 пациенту с криптогенным инсультом, а 220 пациентов наблюдались в контрольной группе[33]. Через 6 месяцев наблюдения ФП была обнаружена у $8,9 \%$ больных с имплантированным устройством и только у 1,4\% пациентов без устройства. Данная технология лучше обнаруживала ФП по сравнению с рутинной практикой (ОР 6,4; 95\% ДИ 1,9-21,7; p <0,001). Частота регистрации случаев ФП в группе вмешательства увеличивалась с длительностью наблюдения. Через 1 месяц ФП регистрировалась в 8 раз чаще, чем в группе контроля. Через 36 месяцев в 10 раз чаще, чем в группе контроля. У большинства больных с имплантируемым устройством (>70\%) ФП была бессимптомной.

Таким образом, в многочисленных работах отмечается, что у пациентов после ИИ для обнаружения бессимптомной и пароксизмальной ФП необходима длительная регистрация сердечного ритма.

\section{Скрининг субклинической фибрилляции предсердий}

Современные имплантируемые устройства способны обнаруживать и сохранять в памяти предсердную тахикардию с высокой частотой любой продолжительности от нескольких секунд до нескольких часов, дней и недель.

Эпизоды частой предсердной тахикардии (ЭЧПТ) $>180$ уд/мин и >5-6 минут относят к субклинической ФП Риск ТЭО будет разным у пациента с одним эпизодом предсердной тахикардии за сутки и с 1000 «пробежками» за сутки. В связи с этим введено понятие «бремя аритмии», которое определяется как суммарное время аритмий за определенный период времени [34].

Через 1,5-2 года после имплантации ЭКС субклиническая ФП была отмечена у 35\% пациентов. Субклиническая ФП была связана с клинической ФП (ОР 5,7 95\% ДИ 4,0-8,0; p <0,001) и повышенным риском ИИ, хотя и несколько меньшим, чем при клинической ФП [35].

В исследовании MOST ЭЧПТ >5 мин ассоциировались с повышенным риском смерти в 2,48 раза (95\%ДИ $1,25-4,91)$ и с увеличением риска ТЭО в 2,79 раза $(95 \%$ ДИ $1,51-5,15)$ [36].

В исследовании «ASSERT» наблюдали за пациентами старше 65 лет с АГ и без ФП $(\mathrm{n}=2580)$. У всех пациентов было имплантированное устройство (кардиостимулятор или дефибриллятор). Через 3 месяца эпизоды предсердной тахикардии $>6$ минут с ЧСС $\geq 190$ ударов наблюдались у $10,1 \%$ пациентов и при долгосрочном наблюдении $(2,5$ года) они были связаны с повышенным риском ФП (ОР 5,56; 95\% ДИ $3,78-8,17 ; \mathrm{p}<0,001)$ и повышенным риском ИИ и ТЭО (ОР 2,49; 95\%ДИ 1,28-4,85; p=0,007) [37].

В исследовании TREND через 30дней после имплантации устройства частота ТЭО была в 2 раза выше при наличии ЭЧПТ >5,5 часов [38].

В настоящее время не существует единого мнения о необходимости назначения пациентам с ЭЧПТ терапии антикоагулянтами, но существует консенсусный документ EHRA, где терапия антикоагулянтом рекомендуется для пациентов $\mathrm{c} \geq 2$ факторами риска по шкале CHA2DS2-VAS ( $\geq 2$ у мужчин, $\geq 3$ у женщин) при бремени ФП $>5.5$ часов в день [34].

Удаленный домашний мониторинг обеспечивает более раннее обнаружение аритмий по сравнению с опросом устройства в условиях больницы. Новые случаи ФП при удаленном мониторинге выявляли раньше, чем при обычном наблюдении (2 дня против 78 дней) [39].

Основываясь на этих данных, домашний мониторинг может быть полезным для быстрой клинической оценки значимости ЭЧПТ и ФП у пациентов с риском развития инсульта и ТЭО.

Рекомендации Европейского общества кардиологов (2016г.) для скрининга на ФП представлены в Таблице 2.

В заключении следует отметить, что альтернативные устройства для диагностики ФП, такие как автоматический прибор для измерения АД с алгоритмом обнаружения ФП, портативные одноканальные регистраторы ЭКГ, смартфоны с возможностью записи ЭКГ являются более эффективными для скрининга ФП, чем пальпация пульса.

Эти устройства можно использовать для оппортунистического и систематического скрининга на ФП.

У пациентов после перенесенного ИИ мониторинг ЭКГ для обнаружения ФП должен быть длительным, вплоть 


\begin{tabular}{|c|c|c|c|}
\hline Рекомендация & Класс & $\begin{array}{l}\text { Уровень } \\
\text { доказанности }\end{array}$ & Ссылки \\
\hline Для установки диагноза ФП необходимо документировать аритмию с помощью ЭКГ & I & $\mathrm{B}$ & {$[40]$} \\
\hline $\begin{array}{l}\text { Для обнаружения ФП у пациентов после преходящей ишемической атаки или ишемическо- } \\
\text { го инсульта рекомендуется регистрация ЭКГ и последующее непрерывное мониторирова- } \\
\text { ние ЭКГ продолжительностью не менее } 72 \text { часов. }\end{array}$ & I & B & {$[43,44]$} \\
\hline $\begin{array}{l}\text { Регулярно выявлять эпизоды тахикардии с высокой частотой на предсердном канале кар- } \\
\text { диостимуляторов и имплантируемых дефибрилляторов, которые должны инициировать } \\
\text { дальнейшее исследование ЭКГ для документирования ФП перед назначением антикоагу- } \\
\text { лянтной терапии. }\end{array}$ & I & B & {$[37,45]$} \\
\hline $\begin{array}{l}\text { Для пациентов с инсультом должен рассматриваться дальнейший мониторинг с исполь- } \\
\text { зованием неинвазивных мониторов или имплантируемых петлевых регистраторов для } \\
\text { выявления бессимптомной ФП }\end{array}$ & IIa & B & {$[33,32]$} \\
\hline $\begin{array}{l}\text { Систематический скрининг ЭКГ для выявления ФП необходим у пациентов > } 75 \text { лет или } \\
\text { тем, кто имеет высокий риск инсульта. }\end{array}$ & IIb & $\mathrm{B}$ & {$[41,25,46]$} \\
\hline
\end{tabular}

до имплантации петлевого регистратора событий.

Важно искать эпизоды субклинической ФП в памяти имплантированных устройств с целью документирования ФП.

В целом, необходимы дальнейшие исследования для уточнения клинической значимости, диагностической ценности и экономической эффективности новых технологий для проведения скрининга на ФП в общей популяции для снижения бремени ФП и ее осложнений.

Disclosures: There is no conflict of interest for all authors.

\section{References}

1. Zoni-Berisso M, Lercari F, Carazza T, Domenicucci S. Epidemiology of atrial fibrillation: European perspective. Clin Epidemiol. 2014; 6:213-220. doi:10.2147/CLEP.S47385.

2. Chugh SS, Havmoeller R, Narayanan K, Singh D, Rienstra M, Benjamin EJ, et all. Worldwide epidemiology of atrial fibrillation: a Global Burden of Disease 2010 Study. Circulation. 2014; 129(8):837-847. doi:10.1161/CIRCULATIONAHA.113.005119.

3. Camm AJ, Kirchhof P, Lip GY, Schotten U, Savelieva I, Ernst S, et all. Guidelines for the management of atrial fibrillation: the Task Force for the Management of Atrial Fibrillation of the European Society of Cardiology (ESC). Eur Heart J. 2010; 31(19):2369-2429. doi:10.1093/eurheartj/ehq278.

4. Murphy A, Banerjee A, Breithardt G, Camm AJ, Commerford P, Freedman B, et all. The World Heart Federation Roadmap for Nonvalvular Atrial Fibrillation. Glob Heart. 2017; 12(4):273-284. doi:10.1016/j.gheart.2017.01.015.

5. Potpara TS, Polovina MM, Marinkovic JM, Lip GY. A comparison of clinical characteristics and long-term prognosis in asymptomatic and symptomatic patients with first-diagnosed atrial fibrillation: the Belgrade Atrial Fibrillation Study. Int $J$ Cardiol. 2013; 168(5):4744-4749. doi:10.1016/j.ijcard.2013.07.234.

6. Friberg L, Engdahl J, Frykman V, Svennberg E, Levin LÅ, Rosenqvist M. Population screening of 75- and 76-year-old men and women for silent atrial fibrillation (STROKESTOP). Europace. 2013; 15(1):135-140. doi:10.1093/europace/eus217.

7. Hart RG, Pearce LA, Aguilar MI. Meta-analysis: antithrombotic therapy to prevent stroke in patients who have nonvalvular atrial fibrillation. Ann Intern Med. 2007; 146(12):857-867. PubMed PMID: 17577005

8. Boriani G, Laroche C, Diemberger I, Fantecchi E, Popescu MI, Rasmussen LH, et all. Asymptomatic atrial fibrillation: clinical correlates, management, and outcomes in the EORP-AF Pilot General Registry. Am JMed. 2015; 128(5):509-518.e2. doi:10.1016/j. amjmed.2014.11.026.

9. Martinez C, Katholing A, Freedman SB. Adverse prognosis of incidentally detected ambulatory atrial fibrillation. A cohort study. Thromb Haemost. 2014; 112(2):276-286. doi: 10.1160/TH4-04-0383.

10. Neubeck L, Orchard J, Lowres N, Freedman SB. To Screen or Not to Screen?Examining the Arguments Against Screening for Atrial Fibrillation. Heart LungCirc. 2017; 26(9):880-886. doi: 10.1016/j.hlc.2017.05.118.

11. 11. Harris K, Edwards D, Mant J. How can we best detect atrial fibrillation? JR Coll Physicians Edinb. 2012 ; 42 Suppl18:5-22. doi:10.4997/JRCPE.2012.S02.Review.

12. Kannel WB, Wolf PA, Benjamin EJ, Levy D. Prevalence, incidence, prognosis, and predisposing conditions for atrial fibrillation: population-based estimates. Am J Cardiol. 1998; 82(8A):2N-9N.PubMedPMID: 9809895.

13. Chamberlain AM, Agarwal SK, Folsom AR, Soliman EZ, Chambless LE, Crow R, et all. A clinical risk score for atrial fibrillation in a biracial prospective cohort (from the Atherosclerosis Risk in Communities [ARIC] study). Am J Cardiol. 2011; 107(1):85-91. doi:10.1016/j.amjcard.2010.08.049.

14. Saliba W, Gronich N, Barnett-Griness O, Rennert G. Usefulness of CHADS2 and CHA2DS2-VASc Scores in the Prediction of 
New-Onset Atrial Fibrillation: A Population-Based Study. Am J Med. 2016; 129(8):843-849. doi:10.1016/j.amjmed.2016.02.029.

15. 15. Taggar JS, Coleman T, Lewis S, Heneghan C, Jones M. Accuracy of methods for diagnosing atrial fibrillation using 12-lead ECG: A systematic review and meta-analysis. Int J Cardiol. 2015; 184:175-183. doi:1016/j.ijcard.2015.02.014.

16. Kirchhof P, Benussi S, Kotecha D, Ahlsson A, Atar D, Casadei B, et all. [2016 ESC Guidelines for the management of atrial fibrillation developed in collaboration with EACTS]. Kardiol Pol. 2016; 74(12):1359-1469. doi:10.5603/KP.2016.0172. Polish.

17. Willits I, Keltie K, Craig J, Sims A. Watch BP Home A for opportunistically detecting atrial fibrillation during diagnosis and monitoring of hypertension: a NICE Medical Technology Guidance. Appl Health Econ Health Policy. 2014; 12(3):255-265. doi: 10.1007/s40258-014-0096-7.

18. Doliwa PS, Rosenqvist M, Frykman V. Paroxysmal atrial fibrillation with silent episodes: intermittent versus continuous monitoring. Scand Cardiovasc J. 2012; 46(3):144-148. doi:10.3109/14017431.2012.661873.

19. Svennberg E, Stridh M, Engdahl J, Al-Khalili F, Friberg L, Frykman V, et all. Safe automatic one-lead electrocardiogram analysis in screening for atrial fibrillation. Europace. 2017; 19(9):1449-1453. doi:10.1093/europace/euw286.

20. Rosenberg MA, Samuel M, Thosani A, Zimetbaum PJ. Use of a noninvasive continuous monitoring device in the management of atrial fibrillation: a pilot study. Pacing Clin Electrophysiol. 2013; 36(3):328-333. doi:10.1111/pace.12053.

21. Mairesse GH, Moran P, Van Gelder IC, Elsner C, Rosenqvist M, Mant J, et all. ESC Scientific Document Group. Screening for atrial fibrillation: a European Heart Rhythm Association (EHRA) consensus document endorsed by the Heart Rhythm Society (HRS), Asia Pacific Heart Rhythm Society (APHRS), and Sociedad Latinoamericana de Estimulación Cardíaca y Electrofisiología (SOLAECE). Europace. 2017; 19 (10):1589-1623. doi:10.1093/europace/eux177.

22. Lowres N, Neubeck L, Salkeld G, Krass I, McLachlan AJ, Redfern J,et all. Feasibility and cost-effectiveness of stroke prevention through community screening for atrial fibrillation using iPhone ECG in pharmacies. The SEARCH-AF study. Thromb Haemost. 2014; 111(6):1167-1176. doi:10.1160/TH14-03-0231.

23. Kaasenbrood F, Hollander M, Rutten FH, Gerhards LJ, Hoes AW, Tieleman RG. Yield of screening for atrial fibrillation in primary care with a hand-held, single-lead electrocardiogram device during influenza vaccination. Europace. 2016; 18(10):1514-1520.

24. Smyth B, Marsden P, Corcoran R, Walsh R, Brennan C, McSharry K, Clarke J, Kelly PJ, Harbison J. Opportunistic screening for atrial fibrillation in a rural area. QJM. 2016; 109(8):539-543. doi:10.1093/qjmed/hcw011.

25. Engdahl J, Andersson L, Mirskaya M, Rosenqvist M. Stepwise screening of atrial fibrillation in a 75-year-old population: implications for stroke prevention. Circulation. 2013; 127(8):930-937. doi:10.1161/CIRCULATIONAHA.112.126656.

26. Proietti M, Mairesse GH, Goethals P, Scavee C, Vijgen J, Blankoff I, et all Belgian Heart Rhythm Week Investigators. A population screening programme for atrial fibrillation: a report from the Belgian Heart Rhythm Week screening programme. Europace. 2016; 18(12):1779-1786. doi:10.1093/europace/euw069.

27. Fitzmaurice DA, Hobbs FD, Jowett S, Mant J, Murray ET, Holder R, et all Screening versus routine practice in detection of atrial fibrillation in patients aged 65 or over: cluster randomized controlled trial. BMJ. 2007; 335(7616):383.

28. Moran PS, Teljeur C, Ryan M, Smith SM. Systematic screening for the detection of atrial fibrillation. Cochrane Database Syst Rev. 2016; (6):CD009586. doi:10.1002/14651858.CD009586.pub3.

29. Hart RG, Diener HC, Coutts SB, Easton JD, Granger CB, O’Donnell MJ, et all; Cryptogenic Stroke/ESUS International Working Group. Embolic strokes of undetermined source: the case for a new clinical construct. Lancet Neurol. 2014; 13(4):429-438. doi: 10.1016/S1474-4422(13)70310-7.

30. Suissa L, Lachaud S, Mahagne MH. Optimal timing and duration of continuous electrocardiographic monitoring for detecting atrial fibrillation in stroke patients. J Stroke Cerebrovasc Dis. 2013; 22(7): 991-995. doi:10.1016/j.jstrokecerebrovasdis.2012.01.015.

31. Wachter R, Weber-Krüger M, Seegers J, Edelmann F, Wohlfahrt J, Wasser K, et all Age-dependent yield of screening for undetected atrial fibrillation in stroke patients: the Find-AF study. J Neurol. 2013; 260(8):2042-2045. doi:10.1007/s00415-013-6935-x.

32. Gladstone DJ, Spring M, Dorian P, Panzov V, Thorpe KE, Hall J, et all; EMBRACE Investigators and Coordinators. Atrial fibrillation in patients with cryptogenic stroke. $N$ Engl J Med. 2014; 370(26):2467-2477. doi:10.1056/NEJMoa1311376.

33. Sanna T, Diener HC, Passman RS, Di Lazzaro V, Bernstein RA, Morillo CA, et all; CRYSTAL AF Investigators. Cryptogenic stroke and underlying atrial fibrillation. N Engl J Med. 2014; 26;370(26):2478-2486. doi:10.1056/NEJMoa1313600.

34. Gorenek B, Bax J, Boriani G, Chen SA, Dagres N, Glotzer TV, et all; ESC Scientific Document Group. Device-detected subclinical atrial tachyarrhythmias: definition, implications and management-an European Heart Rhythm Association (EHRA) consensus document, endorsed by Heart Rhythm Society (HRS), Asia Pacific Heart Rhythm Society (APHRS) and Sociedad Latinoamericana de Estimulación Cardíaca y Electrofisiología (SOLEACE). Europace. 2017; 19(9):1556-1578. doi:10.1093/ europace/eux163.

35. Mahajan R, Perera T, Elliott AD, Twomey DJ, Kumar S, Munwar DA, et all Subclinical device-detected atrial fibrillation and stroke risk: a systematic review and meta-analysis. Eur Heart J. 2018; doi:10.1093/eurheartj/ehx731.

36. Glotzer TV, Hellkamp AS, Zimmerman J, Sweeney MO, Yee R, Marinchak R, et all; MOST Investigators. Atrial high rate episodes detected by pacemaker diagnostics predict death and stroke:report of the Atrial Diagnostics Ancillary Study of the MOde Selection Trial (MOST). Circulation. 2003; 107(12):1614-1619. Epub 2003 Mar 24. PubMed PMID:12668495.

37. Healey JS, Connolly SJ, Gold MR, Israel CW, Van Gelder IC, Capucci A, et all; ASSERT Investigators. Subclinical atrial fibrillation and the risk of stroke. N Engl J Med. 2012; 366(2):120-129. doi:10.1056/NEJMoa1105575.

38. 38. Gonzalez M, Keating RJ, Markowitz SM, Liu CF, Thomas G, Ip JE, et all Newly detected atrial high rate episodes predict long-term mortality outcomes in patients with permanent pacemakers. Heart Rhythm. 2014; 11(12):2214-2221. doi: 10.1016/j. hrthm.2014.08.019.

39. Lorenzoni G, Folino F, Soriani N, Iliceto S, Gregori D. Cost-effectiveness of early detection of atrial fibrillation via remote control of implanted devices. J Eval Clin Pract. 2014; 20(5):570-577. doi: 10.1111/jep.12132.

40. Kirchhof P, Auricchio A, Bax J, Crijns H, Camm J, Diener HC, et all Outcome parameters for trials in atrial fibrillation: executive summary: Recommendations from a consensus conference organized by the German Atrial Fibrillation Competence NETwork 
(AFNET) and the European Heart Rhythm Association (EHRA). EurHeart J. 2007; 28(22):2803-2817.

41. Davis RC, Hobbs FD, Kenkre JE, Roalfe AK, Iles R, Lip GY, et all Prevalence of atrial fibrillation in the general population and in high-risk groups: the ECHOES study. Europace. 2012; 14(11):1553-1559. doi:10.1093/europace/eus087.

42. Lowres N, Neubeck L, Redfern J, Freedman SB. Screening to identify unknown atrial fibrillation. A systematic review. Thromb Haemost. 2013; 110(2):213- 222. doi: 10.1160/TH13-02-0165.

43. Grond M, Jauss M, Hamann G, Stark E, Veltkamp R, Nabavi D, et all. Improved detection of silent atrial fibrillation using 72-hour Holter ECG in patients with ischemic stroke: a prospective multicenter cohort study. Stroke. 2013; 44(12):3357-3364. doi:10.1161/STROKEAHA.113.001884.

44. Rizos T, Güntner J, Jenetzky E, Marquardt L, Reichardt C, Becker R, et all Continuous stroke unit electrocardiographic monitoring versus 24-hour Holter electrocardiography for detection of paroxysmal atrial fibrillation after stroke. Stroke. 2012; 43(10):26892694. Epub 2012 Aug 7. PubMed PMID: 22871678.

45. Israel CW, Grönefeld G, Ehrlich JR, Li YG, Hohnloser SH. Long-term risk of recurrent atrial fibrillation as documented by an implantable monitoring device: implications for optimal patient care. J Am Coll Cardiol. 2004; 43(1):47-52

46. Svennberg E, Engdahl J, Al-Khalili F, Friberg L, Frykman V, Rosenqvist M. Mass Screening for Untreated Atrial Fibrillation: The STROKESTOP Study. Circulation. 2015; 131(25):2176-2184. doi:10.1161/CIRCULATIONAHA.114.014343.

How to cite this article: Zhenisgul Tlegenova, Gulmira Kudaiberdieva, Bekbolat Zholdin, Ayan Abdrakhmanov. Screening for atrial fibrillation [in Russian]. J Clin Med Kaz. 2018; 1(47):18-24 